\title{
Clinical Application of AMH Measurement in Assisted Reproduction
}

\author{
Hang Wun Raymond $\mathrm{Li}^{1 *}$ and Scott M. Nelson ${ }^{2,3,4}$ \\ ${ }^{1}$ Department of Obstetrics and Gynaecology, The University of Hong Kong, Pokfulam, Hong Kong, ${ }^{2}$ School of Medicine, \\ University of Glasgow, Glasgow, Scotland, United Kingdom, ${ }^{3}$ NIHR Bristol Biomedical Research Centre, Bristol, \\ United Kingdom, ${ }^{4}$ The Fertility Partnership, Oxford, United Kingdom
}

OPEN ACCESS

Edited by:

Gedis Grudzinskas, Private Practitioner, London, United Kingdom

Reviewed by: Antonio La Marca, University of Modena and Reggio Emilia, Italy Ilpo Huhtaniemi, Imperial College London, United Kingdom

*Correspondence: Hang Wun Raymond Li raymondli@hku.hk

Specialty section: This article was submitted to Reproduction, a section of the journal Frontiers in Endocrinology

Received: 15 September 2020

Accepted: 30 October 2020

Published: 09 December 2020

Citation:

Li HWR and Nelson SM (2020) Clinical Application of AMH Measurement in Assisted Reproduction. Front. Endocrinol. 11:606744. doi: 10.3389/fendo.2020.606744
Anti-Müllerian hormone reflects the continuum of the functional ovarian reserve, and as such can predict ovarian response to gonadotropin stimulation and be used to individualize treatment pathways to improve efficacy and safety. However, consistent with other biomarkers and age-based prediction models it has limited ability to predict live birth and should not be used to refuse treatment, but rather to inform counselling and shared decision making. The use of absolute clinical thresholds to stratify patient phenotypes, assess discordance and individualize treatment protocols in non-validated algorithms combined with the lack of standardization of assays may result in inappropriate classification and sub-optimal clinical decision making. We propose that holistic baseline phenotyping, incorporating antral follicle count and other patient characteristics is critical. Treatment decisions driven by validated algorithms that use ovarian reserve biomarkers as continuous measures, reducing the risk of misclassification, are likely to improve overall outcomes for our patients.

Keywords: anti-Müllerian hormone, assisted reproduction, in vitro fertilization, intrauterine insemination, ovarian response, gonadotropin dosing

\section{INTRODUCTION}

One major clinical area where anti-Müllerian hormone (AMH) measurement has carved out a clear niche is in assisted reproduction. Over the recent two decades, there have been a growing number of observational studies and randomized controlled trials (RCTs) exploring AMH's role in assessing ovarian reserve, predicting ovarian response and outcomes to treatment, and clarifying its utility for individualizing treatment strategies in women undergoing assisted reproduction treatment (ART) including intrauterine insemination (IUI) with controlled ovarian stimulation or in vitro fertilization (IVF). These clinical applications are based on AMH exhibiting strong correlations with the primordial follicle count (1), the ultimate parameter that represents the conceptual ovarian reserve, as well as the later stages of follicular development that are responsive to gonadotropins and constitute the functional ovarian reserve (2-4). The widespread clinical adoption of AMH has been further enhanced through the ease of scalability of serum/plasma testing, the availability of high precision automated assays, that it can be measured at any part of the menstrual cycle, that small fluctuations observed within and across cycles have been shown not to be clinically important (511 ), and that measurements determined in the months leading up to the index stimulation cycle can 
accurately guide assisted reproduction treatment decisions. In this review, we summarize the recent evidence underpinning the use of $\mathrm{AMH}$ in ART, including how AMH can inform the overall prognostic phenotype and individualize treatment decisions, while highlighting the areas that continue to require further exploration.

\section{INITIAL OBSERVATIONS}

\section{AMH as a Continuous Measure}

The AMH concentration that we receive from that initial blood test is like many biological measures lying on a continuum, from very low at one extreme to very high at the other. However, historically studies have primarily focused on identifying individual threshold values to categorize different types of prognosis and stratify treatments $(12,13)$. In clinical practice it is often helpful to label individuals as having or not having an attribute, such as a potential poor responder, depending on the value of a continuous variable like AMH. However, the dichotomization of continuous variables leads to several problems (14). Importantly much information is lost, so the statistical power and ability to demonstrate an association with the outcome is substantially reduced. Secondly the extent of the variation in outcome between groups may be underestimated, such that individuals close to but on opposite sides of the threshold are characterized as being very different rather than being very similar. Thirdly, using two groups conceals any nonlinearity in the relation between the variable and outcome. Lastly, the use of apparently "optimal" cutpoint (usually that giving the minimum $\mathrm{P}$ value) runs a high risk of a spuriously significant result; the difference in the outcome variable between the groups will be overestimated, perhaps considerably, and the confidence interval will be too narrow. In ART these issues are not unique to $\mathrm{AMH}$ and apply to many routine indices like antral follicle count (AFC), sperm counts, and endometrial thickness which are all continuous measures yet we continue to dichotomize them rather than treat them as continuous variables to enable greater variability in outcomes to be explained. Confirmation of the value of treating $\mathrm{AMH}$ as a continuous measure, enabling greater explanation of variability, has recently been shown in a RCT (15). Simple categorization has a role, but as we become more sophisticated in our understanding of the strengths and weaknesses of $\mathrm{AMH}$ we propose that we should consider $\mathrm{AMH}$ as biology intended-a continuous measure of the functional ovarian reserve.

\section{Ovarian Reserve Markers Should Not Be on the Causal Pathway When Estimating Strength of Associations With Outcomes}

Assessment of the true strength of the correlation between an exposure (such as AMH) and an outcome (for example oocyte yield) requires that the exposure in no way influences the treatment pathway which results in the outcome. Unfortunately for many of the studies that blindly evaluated the correlation of AMH with outcomes $(16,17)$, also measured and acted upon the antral follicle count (AFC), which is itself strongly correlated with $\mathrm{AMH}$ due to the granulosa cells of the smaller antral follicles being the primary source of $\mathrm{AMH}$ (4). By altering the stimulation strategy or dose based on the AFC or other linked factors like age, the researchers will have introduced systematic bias which can lead to an overestimation of the strength of the correlation, which will apply to the primary marker used such as AFC and to a lesser degree the inter-related markers like AMH. The only way the strengths of the association can be truly evaluated is if all patients are treated identically and the researchers are blind to the initial ovarian reserve biomarkers (18). Such a study design is often only seen in randomized controlled trials $(19,20)$, rather than observational studies which are frequently used to assess and report the relative merits of different biomarkers. It is in this context of multicenter large scale RCTs with strictly defined protocols, where a true indication of the performance and limitations of biomarkers such as $\mathrm{AFC}$ and $\mathrm{AMH}$ can be observed.

\section{ROLE OF AMH MEASUREMENT IN IUI WITH CONTROLLED OVARIAN STIMULATION}

Controlled ovarian stimulation and IUI can be a first-line treatment for unexplained infertility as well as infertility due to mild male factor or endometriosis. In this context IUI may commonly be coupled with controlled ovarian stimulation by gonadotropin or oral anti-estrogen drugs which may correct subtle problems of ovulation, slightly increase the number of oocytes available for fertilization, and enhance the accuracy of timing of insemination $(21,22)$.

For AMH to be of value in this context, we anticipate it would identify which patients would benefit from stimulated IUI rather than proceeding directly to IVF, identify an appropriate initial stimulation strategy for example exogenous gonadotropins or aromatase inhibitors, and/or identify the likely prognosis to manage patient expectations regarding the likelihood of success. Unfortunately, although $\mathrm{AMH}$ contributing to all three aspects may be aspirational there have been a limited number of studies evaluating the role of $\mathrm{AMH}$ in IUI management. Historically, studies focused on the association of pretreatment AFC with pregnancy outcomes, with differing conclusions (23-25). The first study on the role of serum $\mathrm{AMH}$ in predicting treatment outcome after ovarian stimulation using gonadotropins was reported in 2010 in 243 women undergoing IUI (26). In this study, baseline AFC was used to alter the dose of starting gonadotropin (ovarian stimulation was achieved with hMG or recombinant FSH starting at $150 \mathrm{IU} /$ day, except for those with $\mathrm{AFC} \geq 10$ or polycystic ovaries, where it was started at 100 and 75 IU/day, respectively). In women who attained live birth either in the first treatment cycle or cumulatively over three treatment cycles, their pretreatment $\mathrm{AMH}$ was significantly higher than those who did not (median AMH $3.47 \mathrm{ng} / \mathrm{ml}$ vs. $2.04 \mathrm{ng} / \mathrm{ml}$ ). Furthermore, $\mathrm{AMH}$ remained a significant predictor on the likelihood of cumulative live birth after controlling for age and body mass 
index of the women in a logistic regression model. Others have subsequently reported similar associations between higher $\mathrm{AMH}$ and higher success rates (27-29). To date validation of optimal treatment strategies (30) or of retrospectively derived algorithms (31) have however been limited and would be the next step for confirmation of a more widespread role of $\mathrm{AMH}$ in stimulated IUI programs.

\section{ROLE OF AMH MEASUREMENT IN IVF}

In IVF program, numerous studies have explored the role of serum $\mathrm{AMH}$ measurement in predicting ovarian response to gonadotropin stimulation, individualizing treatment pathways to improve efficacy and safety, and lastly predicting overall treatment success $(12,13,18,32)$. Given the biological premise of $\mathrm{AMH}$ as a functional ovarian reserve marker, it is not surprising that it is these first two areas where AMH has made the greatest contribution.

\section{Prediction of Suboptimal or Excessive Ovarian Response}

Ovarian stimulation forms an integral part in modern IVF programs. Multiple follicle development and aspiration, and hence collection of multiple oocytes, helps to increase the efficiency of the treatment program. It has been reported that a higher oocyte yield up to around 15 was associated with higher live birth rate in the fresh treatment cycle (33) as well as higher cumulative live birth rate following the fresh and all frozenthawed embryo transfers after one IVF cycle (34).

It is now widely established that $\mathrm{AMH}$ and $\mathrm{AFC}$ are the currently best available predictors of ovarian response and its associated extremes; poor and excessive ovarian response (3537). Accepting the limitations noted above on using observational studies, an individual patient data (IPD) metaanalysis assessing prediction of excessive ovarian response included 57 studies with 4,786 women and concluded that both $\mathrm{AMH}$ and AFC exhibited similar and reasonably good performance in predicting excessive ovarian response in isolation. The area under the receiver operating characteristic (ROC) curve for AMH and AFC was 0.81 and 0.79 respectively (16). Although the combination improved the area under the curve marginally to 0.85 , inclusion of additional covariates such as age or FSH did not improve the prediction further. A second IPD meta-analysis reported on the prediction of poor ovarian response (17). It included data from 28 studies with 5,705 women undergoing IVF treatment. Again, both $\mathrm{AMH}$ and AFC had similar and reasonably good performance in predicting poor ovarian response on their own, with an area under the ROC curve of 0.78 and 0.76 respectively, and once again combining the two or adding age did not significantly improve the prediction. However, as noted for almost all of the studies included in these meta-analyses, AFC was known prior to commencing ovarian stimulation and used to modify the dose which might have led to an overestimation of the strength of the association of AFC.
In the context of predicting suboptimal ovarian response, $\mathrm{AMH}$ and $\mathrm{AFC}$ were among the criteria used to define or predict poor ovarian responders in the Bologna criteria (38) and more recently the Poseidon classification (39). Although the Poseidon criteria has been proposed as an attempt at defining a more homogeneous population, heterogeneity remains. For example, the thresholds for AMH and AFC are not aligned with respect to established correlations between these two indices (4). For treated patients there is no agreed consensus on the nature of the previous stimulation strategy. Lastly patients are dichotomized to either $<35$ or $\geq 35$ years of age, despite the non-linear relationship with oocyte aneuploidy.

An extrapolation of the ability to predict ovarian response is the individualization of the ovarian stimulation regimen in the treatment naïve patient, particularly if aiming for a fresh embryo transfer. In this context achieving an optimal oocyte yield while minimizing the risk of ovarian hyperstimulation syndrome (OHSS) is paramount. Our initial suggestion of AMH-driven algorithms to determine the initial gonadotropin dose and regimen of ovarian stimulation $(40,41)$ have now been confirmed in several RCTs, with the largest $(n=1326)$ assessing the efficacy and safety of follitropin delta (15). In this RCT, the follitropin delta dose was based on the individual women's serum $\mathrm{AMH}$ level and body weight and compared with follitropin alpha at 150 IU with subsequent step up or down according to ovarian response. The two treatment arms had similar mean oocyte yield and live birth rates, and yet the follitropin delta arm had significantly lower rates of suboptimal or excessive response. A recent Cochrane meta-analysis concluded that although individualized dosing of gonadotropin based on ovarian reserve markers might not influence the rate of ongoing pregnancy or live birth compared to standardized dosing, it could reduce the incidence of moderate-to-severe OHSS by prompting the use of a reduced dose of gonadotropin in predicted high responders (42).

As current $\mathrm{AMH}$ assays give different numerical results without any universal standardization $(43,44)$, and that reported studies were performed using different assays, it is not possible to combine the available data to determine cut-offs for predicting excessive or suboptimal ovarian response. Similarly, although historical definitions including the Bologna criteria defined poor ovarian reserve as an AFC of below 5 to 7 , or $\mathrm{AMH}$ level of below $0.5-1.1 \mathrm{ng} / \mathrm{ml}$, while the Poseidon classification adopted an AFC of 5 or AMH of $1.2 \mathrm{ng} / \mathrm{ml}$ as the cut-offs for defining it, it should be noted that these were based on previous studies using different assay methods and hence there may be problems to adopt these apparently simple thresholds universally.

\section{Prediction of Pregnancy or Live Birth in IVF Treatment}

Despite the good performance of $\mathrm{AMH}$ and $\mathrm{AFC}$ in predicting ovarian response, most studies, however, consistently showed that just like age, $\mathrm{AMH}$ and $\mathrm{AFC}$ were poor overall predictors of pregnancy or live birth in the fresh IVF cycle $(17,32,45)$. The summary ROC curves derived from the individual patient data meta-analysis by Broer et al. (17) for prediction of ongoing pregnancy confirmed the limited role of $\mathrm{AMH}, \mathrm{AFC}$ and age, or 
their combinations, with an area under the ROC curve of less than 0.6. Focusing on AMH and live-birth, a meta-analysis on 13 studies found an area under the ROC curve of 0.61 (95\% confidence interval $0.56-0.65$ ) of confirming the limited contribution that $\mathrm{AMH}$ in isolation would have for prognostication of overall livebirth (32).

All these studies were limited by focusing on the rate of pregnancy or live birth in the fresh IVF cycle only. In modernday IVF programs, embryo cryopreservation constitutes an increasingly important part, and hence the cumulative live birth rate from the fresh and all frozen-thawed embryo transfer (FET) cycles combined would be more informative and meaningful than the outcome of the fresh cycle alone (46). A retrospective analysis evaluating the role of baseline AMH in predicting cumulative live birth from the fresh IVF cycle plus all subsequent FET cycles derived from that stimulated cycle was first reported in 2013 (3). It included 1,156 women undergoing the first IVF cycle in a single center treated under the long GnRH agonist protocol or $\mathrm{GnRH}$ antagonist protocol. It suggested that the cumulative live birth rate followed a gradual rising trend with serial increase in serum AMH or AFC over a continuum instead of showing an abrupt change at any threshold value. However, both parameters had only modest performance, which was not better than the women's age alone, in predicting the absolute occurrence of cumulative live birth as demonstrated by the ROC curves (area under the curve being 0.646, 95\% CI 0.616-0.675). After controlling for the women's age and the number of embryos replaced, both serum AMH and AFC were not significant independent predictors of live birth in the fresh IVF cycle nor cumulative live birth suggesting that their association with overall livebirth was through the number of oocytes and thereby number of embryos available to transfer. Another recent study in 9,494 Chinese women similarly demonstrated that increasing $\mathrm{AMH}$ up to $5-7 \mathrm{ng} / \mathrm{ml}$ predicted better cumulative live birth rate in IVF and that it was mainly through the association with oocyte yield (47).

As for women with predicted poor ovarian reserve, a recent retrospective analysis on 825 IVF cycles showed that the live birth rate decreased through Poseidon groups 1, 3, 2, and 4 in order (48). It implies that both AMH or AFC as well as age have an impact on the prediction of live birth. It is worth to note that in the study by Li et al. (3), women with serum AMH $<0.5 \mathrm{ng} / \mathrm{ml}$ still had a cumulative live birth rate of $27 \%$, and cumulative live birth did occur in women with $\mathrm{AMH}$ as low as $0.15 \mathrm{ng} / \mathrm{ml}$. Another secondary analysis on women in the OPTIMIST study, a prospective observational study on 551 women with predicted low prognosis, showed that those in Poseidon 4 group (older women with low ovarian reserve) still had conservative and optimistic cumulative live birth rates of $37 \%$ and $41 \%$ respectively over 18 months of treatment (49).

Collectively all of these data suggest that a patient at any age with a higher AMH has an overall better prognosis. However, due to the limitations of its predictive performance, a threshold value should not be used to deny women from attempting ART, nor to be too pessimistic regarding prognosis based solely on an $\mathrm{AMH}$ value.

\section{Comparing AMH Versus AFC in Predicting Ovarian Response}

Although differences in performance characteristics of $\mathrm{AMH}$ and AFC have been reported in several multi-center RCTs (18), direct head-to-head performance comparison of AMH- or AFC-based dosing algorithms has been more limited. Specifically, two RCTs have compared the performance of a serum AMH or AFC algorithm in predicting ovarian response in an IVF program, with both concluding that there were no significant differences in the proportion of cycles attaining desired ovarian response when the gonadotropin dosing algorithm was determined based on either $\mathrm{AMH}$ or $\operatorname{AFC}(50,51)$. In the first study, 348 Vietnamese women were treated with a long GnRH agonist protocol, and $35.2 \%$ versus $28.4 \%$ of cycles attained the desired response when the AMH-based and AFC-based algorithms were adopted respectively ( $\mathrm{p}>0.05)$, although the incidence of hyper-response was significantly lower in the AMH group (8.6\%) compared to the AFC group (17.4\%) (50). In the second study, 200 participants from Hong Kong were treated on a GnRH antagonist protocol (51). There were no significant differences in the proportion of cycles with desired response between the AMH-based and AFC-based groups (49.0\% versus $54.0 \%$, $\mathrm{p}>0.05$ ), or the number of oocytes retrieved or the follicular output rate. However, significantly more women required an increase in their gonadotropin dose in the $\mathrm{AMH}$ group compared to the AFC group. These findings suggest that clinicians who choose to use these specific published algorithms and treatment strategies would obtain equivalent results whether they use AMH or AFC. However, this conclusion of equivalence does not extend to other untested algorithms or equate to overall equivalence for treatment decision making.

\section{Discordance Between AMH and AFC in Prediction of Ovarian Response}

For most women AMH and AFC will be similar, but discordances can occur with extreme disagreements the most concerning and difficult to interpret clinically. A retrospective analysis on 1,046 women assessed the discordance between $\mathrm{AMH}$ and AFC, by using the $25^{\text {th }}$ and $75^{\text {th }}$ centiles of $\mathrm{AMH}$ (1.4 and $5.3 \mathrm{ng} / \mathrm{ml}$ ) and AFC (6 and 14) respectively as the thresholds (52). In these analyses only 4 patients exhibited a high $\mathrm{AMH}$ but a low AFC and conversely 1 patient exhibited a high AFC and low AMH. Simple categorization may however over emphasize apparent milder discordances, for example in the above study an AMH of $1.3 \mathrm{ng} / \mathrm{ml}$ and an AFC of 7 would be recorded as discordant, but clinically many would perceive as equivalent with a similar response anticipated. In the trial by $\mathrm{Li}$ et al. (51), among the 200 enrolled women, 26.5\% showed discordance between categorization based on AMH or AFC in the pre-treatment cycle $(\kappa=0.560)$, with an overall discordance rate of around $30 \%$. In women who were discordant in $\mathrm{AMH}$ and AFC categories, those having higher AMH within the same AFC quartile had significantly higher oocyte yield and cumulative live birth rate, and the ovarian responsiveness was intermediate between those where $\mathrm{AMH}$ and AFC were concordant on 
either the high or low end (52). Applying to clinical scenarios where $\mathrm{AMH}$ and AFC categories are discordant, it is reasonable to suggest an intermediate dose of gonadotropin between that assigned for the high and low ends. Nonetheless, such a recommendation will require verification in prospective trials.

In view of such discordant scenarios, it has been proposed that a more holistic phenotype which incorporates AMH, AFC and age can be combined into a composite score for the purpose of ovarian response prediction. The ovarian response prediction index (ORPI), calculated as the product of AMH level (ng/ml) and AFC divided by age of the woman (years), was first reported by Oliveira et al. (53). The original study showed that ORPI had good prediction on oocyte yield, and the same group subsequently also showed that using ORPI for individualization of the ovarian stimulation regimen resulted in elimination of OHSS in their center (54). A retrospective analysis on 285 women stimulated with a standardized initiation dose using corifollitropin alpha in the GnRH antagonist protocol confirmed that ORPI was significantly correlated with the oocyte yield (55). ROC curve analysis revealed that the area under the curve for ORPI was comparable to AMH alone and significantly higher than AFC alone for prediction of excessive response, while it was significantly higher than that of $\mathrm{AMH}$ or AFC alone for prediction of suboptimal response. In contrast in the phase II derivation of the follitropin delta algorithm, the inclusion of either or all of age, FSH, or AFC did not increase explanation of the variance by $\geq 5 \%$ above what was initially observed for just bodyweight and AMH. Therefore, although a composite index of ovarian reserve biomarkers may be worth further exploration to try to reduce the unexplained variance in ovarian response in future trials, its overall contribution may be limited and would require systematic and timed scanning.

\section{Timing of AMH Assessment Prior to IVF}

A number of studies have reported inter-cycle fluctuations of $\mathrm{AMH}$ level, and yet the absolute magnitudes of these fluctuations are small and may have limited clinical importance. It was shown that when AMH was measured one month prior to IVF as well as at the start of ovarian stimulation, there was moderate concordance between $\mathrm{AMH}$ categorization measured in the pre-treatment versus the stimulation cycle $(\kappa=0.573)(51)$. Similarly, an analysis of 1326 women in the three months leading up to an index cycle suggested strong correlations $(r=0.92)$, with no systematic variation across the menstrual cycles (56). Others have also shown using different gonadotropins that it can be used in advance of the index cycle for prediction of response $(13,57)$. Hence, although the assessment of AMH can be performed on any day of the cycle in the months preceding ovarian stimulation, as for any response prediction the accuracy will be greatest if it is measured in the index cycle immediately prior to commencing gonadotropins.

\section{Prediction of Embryo Quality}

There are contrasting data reported on the role of $\mathrm{AMH}$ in predicting embryo quality. While some studies revealed that serum AMH was not significantly associated with morphokinetic embryo quality as assessed by time-lapse imaging $(58,59)$, there were reports that the oocyte-specific $\mathrm{AMH}$ concentration in follicular fluid had good prediction on embryonic development and live birth $(60,61)$. It is interesting to further explore the functional relationship of follicular and serum $\mathrm{AMH}$ with oocyte competence, embryo euploidy and its role in embryo selection.

\section{CONCLUDING REMARKS}

$\mathrm{AMH}$ has evolved as a useful tool for the assessment of the functional ovarian reserve and prediction of ovarian response, with performance at least equivalent to or better than AFC. However, just like AFC or age, its ability to predict live birth both in the fresh cycle and cumulatively taking into account all embryos derived from the same index stimulation cycle is limited, and primarily stem from its relationship with the oocyte and hence embryo yield. We propose that we no longer need to debate on which biomarker is best, but rather accept that we can utilize all of the information at our disposal to characterize the baseline phenotype and likely response and modify our treatment strategies accordingly. The use of both $\mathrm{AMH}$ and AFC, as continuous measures, combined with other patient characteristics in validated algorithms will reduce the risk of misclassification and is likely to improve overall outcomes for our patients.

\section{AUTHOR CONTRIBUTIONS}

HL and SN conceived, wrote, and approved the manuscript. All authors contributed to the article and approved the submitted version.

\section{REFERENCES}

1. Hansen KR, Hodnett GM, Knowlton N, Craig LB. Correlation of ovarian reserve tests with histologically determined primordial follicle number. Fertil Steril (2011) 95(1):170-5. doi: 10.1016/j.fertnstert.2010.04.006

2. Nardo LG, Christodoulou D, Gould D, Roberts SA, Fitzgerald CT, Laing I. Anti-Müllerian hormone levels and antral follicle count in women enrolled in in vitro fertilization cycles: relationship to lifestyle factors, chronological age and reproductive history. Gynecol Endocrinol (2007) 23(8):486-93. doi: $10.1080 / 09513590701532815$

3. Li HWR, Lee VCY, Lau EYL, Yeung WSB, Ho PC, Ng EHY. Role of baseline antral follicle count and anti-Mullerian hormone in prediction of cumulative live birth in the first in vitro fertilisation cycle: a retrospective cohort analysis. PLoS One (2013) 8(4):e61095. doi: 10.1371/journal.pone. 0061095

4. Anderson RA, Anckaert E, Bosch E, Dewailly D, Dunlop CE, Fehr D, et al. Prospective study into the value of the automated Elecsys antimüllerian hormone assay for the assessment of the ovarian growing follicle pool. Ferti Steril (2015) 103(4):1074-80.e4. doi: 10.1016/j.fertnstert.2015.01.004 
5. Fanchin R, Schonäuer LM, Righini C, Frydman N, Frydman R, Taieb J. Serum anti-Mullerian hormone dynamics during controlled ovarian hyperstimulation. Hum Reprod (2003) 18:328-32. doi: 10.1093/humrep/deg043

6. La Marca A, Stabile G, Artenisio AC, Volpe A. Serum anti-Mullerian hormone throughout the human menstrual cycle. Hum Reprod (2006) 21 (12):3103-7. doi: 10.1093/humrep/del291

7. Hehenkamp WJK, Looman CWN, Themmen APN, de Jong FH, Te Velde ER, Broekmans FJM. Anti-Mullerian hormone levels in the spontaneous menstrual cycle do not show substantial fluctuation. J Clin Endocrinol Metab (2006) 91:4057-63. doi: 10.1210/jc.2006-0331

8. Streuli I, Fraisse T, Pillet C, Ibecheole V, Bischof P, de Ziegler D. Serum antimüllerian hormone levels remain stable throughout the menstrual cycle and after oral or vaginal administration of synthetic sex steroids. Fertil Steril (2008) 90(2):395-400. doi: 10.1016/j.fertnstert.2007.06.023

9. van Disseldorp J, Lambalk CB, Kwee J, Looman CW, Eijkemans MJ, Fauser $\mathrm{BC}$, et al. Comparison of inter- and intra-cycle variability of anti-Mullerian hormone and antral follicle counts. Hum Reprod (2010) 25(1):221-7. doi: 10.1093/humrep/dep366

10. Sowers M, McConnell D, Gast K, Zheng H, Nan B, McCarthy JD, et al. AntiMüllerian hormone and inhibin B variability during normal menstrual cycles. Fertil Steril (2010) 94(4):1482-6. doi: 10.1016/j.fertnstert.2009.07.1674

11. Gracia CR, Shin SS, Prewitt M, Chamberlin JS, Lofaro LR, Jones KL, et al. Multi-center clinical evaluation of the Access AMH assay to determine AMH levels in reproductive age women during normal menstrual cycles. J Assist Reprod Genet (2018) 35(5):777-83. doi: 10.1007/s10815-018-1141-5

12. Dewailly D, Andersen CY, Balen A, Broekmans F, Dilaver N, Fanchin R, et al. The physiology and clinical utility of anti-Mullerian hormone in women. Hum Reprod Update (2014) 20(3):370-85. doi: 10.1093/humupd/dmt062

13. La Marca A, Sunkara SK. Individualization of controlled ovarian stimulation in IVF using ovarian reserve markers: from theory to practice. Hum Reprod Update (2014) 20(1):124-40. doi: 10.1093/humupd/dmt037

14. Altman DG, Royston $P$. The cost of dichotomising continuous variables. $B M J$ (2006) 332(7549):1080. doi: 10.1136/bmj.332.7549.1080

15. Andersen AN, Nelson SM, Fauser BC, García-Velasco JA, Klein BM, Arce JC. ESTHER-1 study group. Individualized versus conventional ovarian stimulation for in vitro fertilization: a multicenter, randomized, controlled, assessor-blinded, phase 3 noninferiority trial. Fertil Steril (2017) 107(2):38796.e4. doi: 10.1016/j.fertnstert.2016.10.033

16. Broer SL, Dólleman M, van Disseldorp J, Broeze KA, Opmeer BC, Bossuyt PM, et al. IPD-EXPORT Study Group. Prediction of an excessive response in in vitro fertilization from patient characteristics and ovarian reserve tests and comparison in subgroups: an individual patient data meta-analysis. Fertil Steril (2013) 100(2):420-9.e7. doi: 10.1016/j.fertnstert.2013.04.024

17. Broer SL, van Disseldorp J, Broeze KA, Dolleman M, Opmeer BC, Bossuyt P, et al. IMPORT study group. Added value of ovarian reserve testing on patient characteristics in the prediction of ovarian response and ongoing pregnancy: an individual patient data approach. Hum Reprod Update (2013) 19(1):26-36. doi: 10.1093/humupd/dms041

18. Iliodromiti S, Anderson RA, Nelson SM. Technical and performance characteristics of anti-Müllerian hormone and antral follicle count as biomarkers of ovarian response. Hum Reprod Update (2015) 21(6):698-710. doi: 10.1093/humupd/dmu062

19. Andersen AN, Witjes H, Gordon K, Mannaerts B. Xpect investigators. Predictive factors of ovarian response and clinical outcome after IVF/ICSI following a $\mathrm{rFSH} / \mathrm{GnRH}$ antagonist protocol with or without oral contraceptive pretreatment. Hum Reprod (2011) 26(12):3413-23. doi: 10.1093/humrep/der318

20. Nelson SM, Klein BM, Arce JC. Comparison of antimüllerian hormone levels and antral follicle count as predictor of ovarian response to controlled ovarian stimulation in good-prognosis patients at individual fertility clinics in two multicenter trials. Fertil Steril (2015) 103(4):923-30.e1. doi: 10.1016/j.fertnstert.2014.12.114

21. Cantineau AE, Cohlen BJ, Heineman MJ. Ovarian stimulation protocols (antioestrogens, gonadotrophins with and without GnRH agonists/antagonists) for intrauterine insemination (IUI) in women with subfertility. Cochrane Database Syst Rev (2007) 2):CD005356. doi: 10.1002/14651858.CD005356.pub2

22. van Rumste MME, Custers IM, van der Veen F, van Wely M, Evers JLH, Mol BWJ. The influence of the number of follicles on pregnancy rates in intrauterine insemination with ovarian stimulation: a meta-analysis. Hum Reprod Update (2008) 14(6):563-70. doi: 10.1093/humupd/dmn034
23. Chang MY, Chiang CH, Chiu TH, Hsieh TT, Soong YK. The antral follicle count predicts the outcome of pregnancy in a controlled ovarian hypertimulation/intrauterine insemination program. J Assist Reprod Genet (1998) 15(1):12-7. doi: 10.1023/a:1022518103368

24. Erdem M, Erdem A, Guler I, Atmaca S. Role of antral follicle count in controlled ovarian hyperstimulation and intrauterine insemination cycles in patients with unexplained subfertility. Fertil Steril (2008) 90(2):360-6. doi: 10.1016/j.fertnstert.2007.06.028

25. Ng EHY, Yeung WSB, Ho PC. The significance of antral follicle count in controlled ovarian stimulation and intrauterine insemination. J Assist Reprod Genet (2005) 22:323-8. doi: 10.1007/s10815-005-6785-2

26. Li HWR, Yeung WSB, Lau EYL, Ho PC, Ng EHY. Evaluating the performance of serum antimullerian hormone concentration in predicting the live birth rate of controlled ovarian stimulation and intrauterine insemination. Fertil Steril (2010) 94(6):2177-81. doi: 10.1016/j.fertnstert.2009.12.059

27. Wang MH, Chen $\mathrm{CH}$, Wang CW, Hsu MI, Tzeng CR. A higher anti-Müllerian hormone level is associated with an increased chance of pregnancy in patients undergoing controlled ovarian stimulation and intrauterine insemination. J Obstet Gynaecol (2015) 35(1):64-8. doi: 10.3109/01443615. 2014.935718

28. Bakas P, Boutas I, Creatsa M, Vlahos N, Gregoriou O, Creatsas G, et al. Can anti-Mullerian hormone (AMH) predict the outcome of intrauterine insemination with controlled ovarian stimulation? Gynecol Endocrinol (2015) 31(10):765-8. doi: 10.3109/09513590.2015.1025381

29. Moro F, Tropea A, Scarinci E, Leoncini E, Boccia S, Federico A, et al. AntiMüllerian hormone concentrations and antral follicle counts for the prediction of pregnancy outcomes after intrauterine insemination. Int $J$ Gynaecol Obstet (2016) 133(1):64-8. doi: 10.1016/j.ijgo.2015.08.021

30. Vaiarelli A, Drakopoulos P, Blockeel C, De Vos M, van de Vijver A, Camus M, et al. Limited ability of circulating anti-Müllerian hormone to predict dominant follicular recruitment in PCOS women treated with clomiphene citrate: a comparison of two different assays. Gynecol Endocrinol (2016) 32 (3):227-30. doi: 10.3109/09513590.2015.1110138

31. Di Paola R, Garzon S, Giuliani S, Lagana AS, Noventa M, Parissone F, et al. Are we choosing the correct FSH starting dose during controlled ovarian stimulation for intrauterine insemination cycles? Potential application of a nomogram based on woman's age and markers of ovarian reserve. Gynecol Obstet (2018) 298(5):1029-35. doi: 10.1007/s00404-018-4906-2

32. Iliodromiti S, Kelsey TW, Wu O, Anderson RA, Nelson SM. The predictive accuracy of anti-Müllerian hormone for live birth after assisted conception: a systematic review and meta-analysis of the literature. Hum Reprod Update (2014) 20(4):560-70. doi: 10.1093/humupd/dmu003

33. Sunkara SK, Rittenberg V, Raine-Fenning N, Bhattacharya S, Zamora J, Coomarasamy A. Association between the number of eggs and live birth in IVF treatment: an analysis of 400135 treatment cycles. Hum Reprod (2011) 26 (7):1768-74. doi: 10.1093/humrep/der106

34. Smith ADAC, Tilling K, Nelson SM, Lawlor DA. Live-birth rate associated with repeat in vitro fertilization treatment cycles. JAMA (2015) 314(24):265462. doi: 10.1001/jama.2015.17296

35. Broekmans FJ, Kwee J, Hendriks DJ, Mol BW, Lambalk CB. A systematic review of tests predicting ovarian reserve and IVF outcome. Hum Reprod Update (2006) 12:685-718. doi: 10.1093/humupd/dml034

36. Broer SL, Mol B, Dolleman M, Fauser BC, Broekmans FJM. The role of antiMullerian hormone assessment in assisted reproductive technology outcome. Curr Opin Obstet Gynecol (2010) 22:193-201. doi: 10.1097/GCO.0b013e3283384911

37. Broer SL, Dólleman M, Opmeer BC, Fauser BC, Mol BW, Broekmans FJ. $\mathrm{AMH}$ and $\mathrm{AFC}$ as predictors of excessive response in controlled ovarian hyperstimulation: a meta-analysis. Hum Reprod Update (2011) 17(1):46-54. doi: 10.1093/humupd/dmq034

38. Ferraretti AP, La Marca A, Fauser BC, Tarlatzis B, Nargund G, Gianaroli L. ESHRE working group on Poor Ovarian Response Definition. ESHRE consensus on the definition of 'poor response' to ovarian stimulation for in vitro fertilization: the Bologna criteria. Hum Reprod (2011) 26(7):1616-24. doi: 10.1093/humrep/der092

39. Alviggi C, Andersen CY, Buehler K, Conforti A, De Placido G, Esteves SC, et al. A new more detailed stratification of low responders to ovarian stimulation: from a poor ovarian response to a low prognosis concept. Fertil Steril (2016) 105:1452-3. doi: 10.1016/j.fertnstert.2016.02.005 
40. Nelson SM, Yates RW, Fleming R. Serum anti-Mullerian hormone and FSH: prediction of live birth and extremes of response in stimulated cyclesimplications for individualization of therapy. Hum Reprod (2007) 22 (9):2414-21. doi: 10.1093/humrep/dem204

41. Nelson SM, Anderson RA, Broekmans FJ, Raine-Fenning N, Fleming R, La Marca A. Anti-Mullerian hormone: clairvoyance or crystal clear? Hum Reprod (2012) 27(3):631-6. doi: 10.1093/humrep/der446

42. Lensen SF, Wilkinson J, Leijdekkers JA, La Marca A, Mol BWJ, Marjoribanks $\mathrm{J}$, et al. Individualised gonadotropin dose selection using markers of ovarian reserve for women undergoing in vitro fertilisation plus intracytoplasmic sperm injection (IVF/ICSI). Cochrane Database Syst Rev (2018) 2:CD012693. doi: 10.1002/14651858.CD012693.pub2

43. Li HWR, Wong BPC, Ip WK, Yeung WSB, Ho PC, Ng EHY. Comparative evaluation of three new commercial immunoassays for anti-Müllerian hormone measurement. Hum Reprod (2016) 31(12):2796-802. doi: 10.1093/ humrep/dew248

44. Iliodromiti S, Salje B, Dewailly D, Fairburn C, Fanchin R, Fleming R, et al. Non-equivalence of anti-Müllerian hormone automated assays-clinical implications for use as a companion diagnostic for individualised gonadotrophin dosing. Hum Reprod (2017) 32(8):1710-5. doi: 10.1093/ humrep/dex219

45. Broer SL, Mol BWJ, Hendriks D, Broekmans FJM. The role of antimullerian hormone in prediction of outcome after IVF: comparison with the antral follicle count. Fertil Steril (2009) 91(3):705-14. doi: 10.1016/j.fertnstert.2007.12.013

46. Borini A, Cattoli M, Bulletti C, Goticchio G. Clinical efficiency of oocyte and embryo cryopreservation. Ann N Y Acad Sci (2008) 1127:49-58. doi: 10.1196/ annals. 1434.012

47. Hu KL, Liu FT, Xu H, Li R, Qiao J. Association of serum anti-Müllerian hormone and other factors with cumulative live birth rate following IVF. Reprod BioMed Online (2020) 40(5):675-83. doi: 10.1016/j.rbmo.2020.01.024

48. Abdullah RK, Liu N, Zhao Y, Shuang Y, Shen Z, Zeng H, et al. Cumulative live-birth, perinatal and obstetric outcomes for POSEIDON groups after IVF/ ICSI cycles: a single-center retrospective study. Sci Rep (2020) 10(1):11822. doi: 10.1038/s41598-020-68896-1

49. Leijdekkers JA, Eijkemans MJC, van Tilborg TC, Oudshoorn SC, van Golde RJT, Hoek A, et al. OPTIMIST study group. Cumulative live birth rates in lowprognosis women. Hum Reprod (2019) 34(6):1030-41. doi: 10.1093/humrep/ dez051

50. Lan VT, Linh NK, Tuong HM, Wong PC, Howles CM. Anti-Müllerian hormone versus antral follicle count for defining the starting dose of FSH. Reprod BioMed Online (2013) 27(4):390-9. doi: 10.1016/j.rbmo.2013.07.008

51. Li HWR, Ko JKY, Lee VCY, Yung SSF, Lau EYL, Yeung WSB, et al. Comparison of antral follicle count and serum anti Müllerian hormone level for determination of gonadotropin dosing in in-vitro fertilization: randomized trial. Ultrasound Obstet Gynecol (2020) 55(3):303-9. doi: 10.1002/uog.20402

52. Li HWR, Lee VCY, Lau EYL, Yeung WSB, Ho PC, Ng EHY. Ovarian response and cumulative live birth rate of women undergoing in-vitro fertilisation who had discordant anti-Mullerian hormone and antral follicle count measurements: a retrospective study. PLoS One (2014) 9(10):e108493. doi: 10.1371/journal.pone.0108493

53. Oliveira JBA, Baruffi RLR, Petersen CG, Mauri AL, Nascimento AM, Vagnini $\mathrm{L}$, et al. A new ovarian response prediction index (ORPI): implications for individualized controlled ovarian stimulation. Reprod Biol Endocrinol (2012) 10:94. doi: 10.1186/1477-7827-10-94

54. Oliveira JBA, Franco JG. The ovarian response prediction index (ORPI) as a clinical internal quality control to prevent ovarian hyperstimualtion syndrome. JBRA Assist Reprod (2016) 20(3):91-2. doi: 10.5935/15180557.20160021

55. Ng DYT, Ko JKY, Li HWR, Lau EYL, Yeung WSH, Ho PC, et al. Performance of ovarian response prediction index (ORPI) in predicting ovarian response and livebirth in the in-vitro fertilisation cycle using a standard stimulation with corifollitropin alpha in a GnRH antagonist protocol. Hum Fertil (2020). doi: 10.1080/14647273.2020.1805517

56. Nelson SM, Larsson P, Mannaerts BMJL, Nyboe Andersen A, Fauser BCJM. Anti-Müllerian hormone variability and its implications for the number of oocytes retrieved following individualized dosing with follitropin delta. Clin Endocrinol (Oxf) (2019) 90(5):719-26. doi: 10.1111/cen.13956

57. Neves AR, Blockeel C, Griesinger G, Garcia-Velasco JA, La Marca A, Rodriguez I, et al. The performance of the Elecsys ${ }^{\circledR}$ anti-Müllerian hormone assay in predicting extremes of ovarian response to corifollitropin alfa. Reprod BioMed Online (2020) 41(1):29-36. doi: 10.1016/j.rbmo.2020.03.023

58. Alexopoulou E, Pinborg A, Budtz-Jørgensen E, Zedeler A. Comparing early embryo morphokinetics with time-lapse microscopy in patients with low and normal ovarian response to ovarian stimulation. Reprod Biol (2019) 19:12732. doi: 10.1016/j.repbio.2019.03.002

59. Korkidakis A, Cho KK, Albert A, Au J, Mellon J, Dunne CM. Anti-Müllerian hormone and embryo quality as determined by time-lapse imaging. Minerva Ginecol (2020) 72(3):132-7. doi: 10.23736/S0026-4784.20.04546-3

60. O'Brien Y, Wingfield M, O'Shea LC. Anti-Müllerian hormone and progesterone levels in human follicular fluid are predictors of embryonic development. Reprod Biol Endocrinol (2019) 17(1):47. doi: 10.1186/s12958019-0492-9

61. Ciepiela P, Dulęba AJ, Kario A, Chełstowski K, Branecka-Woźniak D, Kurzawa R. Oocyte matched follicular fluid anti-Müllerian hormone is an excellent predictor of live birth after fresh single embryo transfer. Hum Reprod (2019) 34(11):2244-53. doi: 10.1093/humrep/dez186

Conflict of Interest: SN reports personal fees from Access Fertility, personal fees and non-financial support from Ferring, personal fees and non-financial support from Merck, personal fees from Coopers Genomics, personal fees and nonfinancial support from Ferring Pharmaceuticals, personal fees from The Fertility Partnership, grants, personal fees and non-financial support from Roche Diagnostics, personal fees from Modern Fertility, other from Delivery I, outside the submitted work.

The remaining author declares that the research was conducted in the absence of any commercial or financial relationships that could be construed as a potential conflict of interest.

Copyright $(2020$ Li and Nelson. This is an open-access article distributed under the terms of the Creative Commons Attribution License (CC BY). The use, distribution or reproduction in other forums is permitted, provided the original author(s) and the copyright owner(s) are credited and that the original publication in this journal is cited, in accordance with accepted academic practice. No use, distribution or reproduction is permitted which does not comply with these terms. 